\title{
Hepatitis E virus in patients with acute hepatitis in Cape Town, South Africa, 2011
}

\author{
S Korsman, ${ }^{1} \mathrm{MB}$ ChB, MMed (Virology), FCPath (SA) Viro; D Hardie, ${ }^{1}$ MB ChB, MMed (Virology); M Kaba, ${ }^{2} \mathrm{MD}, \mathrm{PhD}$ \\ ${ }^{1}$ National Health Laboratory Service, Groote Schuur Hospital, Cape Town, and Division of Medical Virology, Department of Pathology, \\ Faculty of Health Sciences, University of Cape Town, South Africa \\ ${ }^{2}$ Division of Medical Microbiology, Department of Pathology, and Institute of Infectious Disease and Molecular Medicine, Faculty of Health Sciences, \\ University of Cape Town, South Africa
}

Corresponding author: S Korsman (stephen.korsman@nhls.ac.za)

Background. Early hepatitis E virus (HEV) seroprevalence studies in South Africa (SA) showed seroprevalence rates of 2 - $10 \%$, and suggested waterborne transmission. More recent studies in Cape Town, SA, reported HEV seroprevalence rates of $28 \%$ and $26 \%$ in outpatients without liver disease and blood donors, respectively. An association was found with eating pork or bacon/ham. Only 3 human cases of hepatitis $\mathrm{E}$ in SA have been reported in the literature.

Objectives. To find evidence of HEV infection in hospitalised patients with acute hepatitis and no other identified cause.

Methods. Leftover serum samples were retrieved for patients negative for hepatitis viruses A, B and C, where no other cause of hepatitis was identified. Samples were tested for HEV by polymerase chain reaction (PCR) and IgM and IgG enzyme-linked immunosorbent assay (ELISA). Results. Anti-HEV IgG was detected in 39/132 specimens (29.5\%; 95\% confidence interval (CI) 22.4 - 37.8 ), and anti-HEV IgM in $2 / 125$ specimens (1.6\%; 95\% CI 0.4 - 5.7). No specimen tested positive by PCR.

Conclusions. IgG seroprevalence found in this study was similar to that previously reported in Cape Town. IgM positivity in 2 patients was not confirmed by PCR. Locally, hepatitis E may not be a common cause of clinically apparent hepatitis that requires hospitalisation.

S Afr Med J 2019;109(8):582-583. DOI:10.7196/SAMJ.2019.v109i8.13867

Hepatitis E virus (HEV) is a common cause of acute hepatitis worldwide. Genotypes 1 and 2 are associated with waterborne transmission and faecal-oral spread, while genotypes 3 and 4 are associated with zoonotic transmission by meat from pigs and other animals. ${ }^{[1]}$

Early HEV seroprevalence studies in South Africa (SA) showed seroprevalence rates of $2.05 \%$ in Gauteng ${ }^{[2]}$ and $10.7 \%$ in the Eastern and Western Cape provinces. ${ }^{[3]}$ The latter study suggested waterborne transmission (odds ratio (OR) 2.85) ${ }^{[3]}$ Recent studies in Cape Town (the Western Cape) reported HEV seroprevalence rates of $28 \%$ and $26 \%$ in patients without liver disease attending clinics at teaching hospitals ${ }^{[1]}$ and in blood donors, ${ }^{[4]}$ respectively. Both studies showed an increase in seroprevalence with age. ${ }^{[1,4]}$ Madden et al. ${ }^{[1]}$ found an association with eating pork (OR 1.93; 95\% confidence interval (CI) 1.45 - 2.55) or bacon/ham (OR 1.50; 95\% CI 1.14 - 1.98). Lopes et al. ${ }^{[4]}$ showed that HEV seroprevalence did not correlate with hepatitis A seroprevalence, and had different age and socioeconomic associations, suggesting an infection route other than waterborne transmission. ${ }^{[4]} \mathrm{HEV}-3$ circulates in humans and animals in SA. Three human cases of HEV-3 have been reported in Cape Town. ${ }^{[1,5,6]}$ Two studies reported circulation of HEV-3 in pigs in the Eastern and Western Cape. ${ }^{[7,8]}$

We investigated evidence of HEV infection in hospitalised patients with acute hepatitis at Groote Schuur Hospital, Cape Town.

\section{Methods}

Over a 60-day period, serum specimens submitted to the diagnostic laboratory at the National Health Laboratory Service, Groote Schuur Hospital, were identified with raised transaminases, negative serology for acute hepatitis A, B and C, and no other identifiable cause in further investigations on the patients of origin. Results for all serum specimens tested serologically for hepatitis A, B and C were extracted from the laboratory information system $(N=2970)$. Only those that tested negative for all three infections were selected $(n=552)$, and were narrowed down to specimens with raised aspartate aminotransferase (AST) and alanine aminotransferase (ALT) $(n=478)$. Additional laboratory samples were screened for other identified causes, leaving 391 without a known cause. Finally, 164 with sufficient specimen remaining $(550 \mu \mathrm{L})$ were included in the study.

All specimens were tested by reverse transcription polymerase chain reaction (PCR) capable of detecting all 4 relevant genotypes, and by enzyme-linked immunosorbent assay (ELISA). IgM and IgG ELISAs were performed using Fortress Diagnostics HEV-IgM and HEV-IgG ELISA kits (Fortress Diagnostics, UK) as per manufacturer's protocol. For molecular detection, nucleic acid from $500 \mu \mathrm{L}$ serum was eluted into $50 \mu \mathrm{L}$ using Nuclisens EasyMag (bioMerieux, the Netherlands). Onestep reverse transcription PCR was performed with $10 \mu \mathrm{L}$ nucleic acid eluate using SuperScript One-Step RT-PCR System with Platinum Taq (Thermo Fisher Scientific, USA) in $50 \mu \mathrm{L}$ final volume. Nested PCR was performed with $2 \mu \mathrm{L}$ prenested amplicon in $50 \mu \mathrm{L}$ final volume using SuperTherm Taq DNA Polymerase (Separation Scientific, South Africa). Primers (forward outer 3156, reverse outer 3157, forward inner 3158, reverse inner 3159) and cycling conditions were as described by Meng et al. ${ }^{[9]}$ Products (348 base pairs) were viewed under ultraviolet light on $2 \%$ agarose gel.

\section{Ethical approval}

The study was approved by the Human Research Ethics Committee of the University of Cape Town (ref. no. 074/2012).

\section{Results}

Patient demographics in the laboratory information system were limited. Age of participants ranged from 3 to 88 (median 34) years. Eighty-nine participants were females and 75 were males. 


\begin{tabular}{|c|c|c|c|}
\hline Results & Anti-HEV IgG & Anti-HEV IgM & HEV PCR \\
\hline Positive & $39(29.5 \%)$ & $2^{\star}(1.6 \%)$ & 0 \\
\hline Negative & 93 & 123 & 164 \\
\hline Total & $132^{+}$ & $125^{\dagger}$ & 164 \\
\hline
\end{tabular}

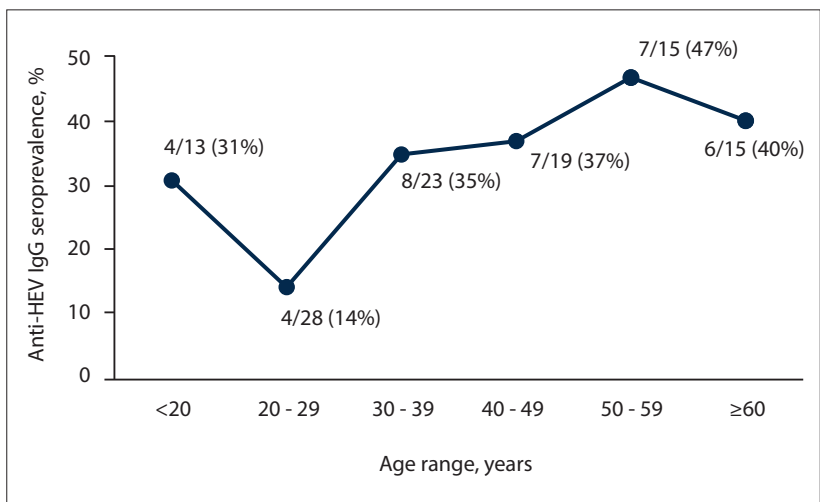

Fig. 1. Hepatitis E IgG seroprevalence by age for participants with a recorded age. Number of positive samples per age group is shown, with total number of samples per age group, and the seroprevalence percentage. (HEV = hepatitis $E$ virus.)

The test results are shown in Table 1. Anti-HEV IgG was detected in $39 / 132$ specimens $(29.5 \%$; 95\% CI 22.4 - 37.8), and anti-HEV IgM in $2 / 125$ specimens $(1.6 \%$; $95 \%$ CI 0.4 - 5.7), both of which were also IgG positive. Both positive IgM results were near the cut-off (optical densities: 0.651 and 0.283 ; both below the 2.5 times cut-off optical density of 0.268 ), suggesting possible nonspecific ELISA reactivity. Fig. 1 shows the IgG seroprevalence curve for participants with known ages. No specimen tested positive by PCR.

\section{Discussion}

This study was the first to investigate HEV infection in patients with acute hepatitis in SA. IgG seroprevalence (29\%) found in this study was similar to IgG seroprevalence rates reported in Cape Town. ${ }^{[1,4]}$ Although the sample size was small, the same trend of increasing seroprevalence with age, with a significant increase in patients $>30$ years, can be seen, as in the larger study on patients without liver disease (Fig. 1). ${ }^{[1]}$ The anomalous higher seroprevalence in patients $<20$ years of age may be an artefact due to the small sample sizes being compared. The lack of PCR-confirmed acute hepatitis E in this study may be due to selection bias, as HEV often causes a mild illness, with most patients not requiring blood tests to investigate clinically apparent acute hepatitis. The 2 IgM low-positive specimens in this study may reflect acute HEV infection after clearance of viraemia, but may also reflect the known nonspecificity problems of IgM assays in populations of ill, hospitalised patients. ${ }^{[10]}$ The sensitivity and specificity for the IgM kit used are reported in the package insert as $97.1 \%$ and $100 \%$, respectively.

\section{Study limitations}

The main limitation of this study is specimen selection bias, as we only investigated patients tested for hepatitis A, B and C. We did not investigate HEV co-infection with other hepatitis viruses. Other limitations and potential biases include sampling of only public healthcare-sector patients, sample size and lack of patient follow-up by researchers. The findings in this study may not apply to other provinces, which may have a different HEV epidemiology.

\section{Conclusions}

HEV is present in the Western Cape, and is associated with consumption of food derived from pig meat; ${ }^{[1]}$ however, locally it may not be a common cause of clinically apparent hepatitis that requires hospitalisation. Further studies aimed at detecting acute HEV infection, both severe and mild, are warranted to elucidate the clinical picture of HEV infection in the Western Cape and SA.

\section{Declaration. None.}

\section{Acknowledgements. None.}

Author contributions. MK and DH: study outline, manuscript review; SK: study design, sample collection, laboratory work, analysis, manuscript writing/review.

Funding. We thank the Poliomyelitis Research Foundation for funding this study (grant 12/11). MK was a Wellcome Trust (UK) fellow (102429/Z/13/Z) and is currently supported by the Carnegie Corporation of New York (USA) early-career fellowship, the Canadian Institutes for Health Research (CIHR), Canadian HIV Trials Network (CTN) international fellowship, and the US National Institutes of Health (1R01HD093578-01).

Conflicts of interest. None.

\footnotetext{
1. Madden RG, Wallace S, Sonderup M, et al. Hepatitis E virus: Western Cape, South Africa. World J Gastroenterol 2016;22(44):9853-9859. https://doi.org/10.3748/wjg.v22.i44.9853

2. Grabow WO, Favorov MO, Khudyakova NS, Taylor MB, Fields HA. Hepatitis E seroprevalence in 2. Grabow WO, Favorov MO, Khudyakova NS, Taylor MB, Fields HA. Hepatitis E seroprevalence in
selected individuals in South Africa. J Med Virol 1994;44(4):384-388. https://doi.org/10.1002/jmv. 1890440412

3. Tucker TJ, Kirsch RE, Louw SJ, Isaacs S, Kannemeyer J, Robson SC. Hepatitis E in South Africa: Evidence for sporadic spread and increased seroprevalence in rural areas. J Med Virol 1996;50(2):117-119. https://doi.org/10.1002/(SICI) 1096-9071(199610)50:2<117::AID-JMV3>3.0.CO;2-D

4. Lopes T, Cable R, Pistorius C, et al. Racial differences in seroprevalence of HAV and HEV in blood donors in the Western Cape, South Africa: A clue to the predominant HEV genotype? Epidemiol Infect 2017;145(9):1910-1912. https://doi.org/10.1017/S0950268817000565

5. Andersson MI, Preiser W, Maponga TG, et al. Immune reconstitution hepatitis E: A neglected complication of antiretroviral therapy in Africa? AIDS 2013;27(3):487-489. https://doi.org/10.1097/ QAD.0b013e32835b1074

6. Andersson MI, Stead PA, Maponga T, van der Plas H, Preiser W. Hepatitis E virus infection: An underdiagnosed infection in transplant patients in southern Africa? J Clin Virol 2015;70:23-25. https://doi. org $/ 10.1016 /$ jijon in transp

7. Adelabu OA, Iweriebor BC, Nwodo UU, Obi LC, Okoh AI. Incidence and molecular characterization of hepatitis E virus from swine in Eastern Cape, South Africa. Adv Virol 2017;2017:1073253. https:// of hepatitis E virus from swine
doi.org/10.1155/2017/1073253

8. Van Helden L, Korsman S, Grewar J, et al. A One Health approach to investigation of zoonotic hepatitis $\mathrm{E}$ virus in Cape Town, South Africa. In: 4th International One Health Congress and 6th Biennial Congress of the International Association for Ecology and Health, 3 - 7 December 2016, Melbourne, Australia. https://link.springer.com/article/10.1007/s10393-016-1191-z (accessed 19 June 2019).

9. Meng XJ, Purcell RH, Halbur PG, et al. A novel virus in swine is closely related to the human hepatitis E virus. Proc Natl Acad Sci USA 1997;94(18):9860-9865. https://doi.org/10.1073/pnas.94.18.9860

10. Abravanel F, Chapuy-Regaud S, Lhomme S, et al. Performance of anti-HEV assays for diagnosing acute hepatitis E in immunocompromised patients. J Clin Virol 2013;58(4):624-628. https://doi. org/10.1016/j.jcv.2013.10.003
}

Accepted 4 February 2019 